\section{Toward Applications of Ceramic Nanostructures}

\section{Sudipta Seal and Marie-Isabelle Baraton, Guest Editors}

\begin{abstract}
This article serves to introduce the January 2004 issue of MRS Bulletin on progress toward applications of ceramic nanostructures. Conventional ceramic materials are widely used today in areas ranging from structural to biological applications, and in devices as diverse as lasers, semiconductors, sensors, and piezoelectric components. Such materials include oxides, carbides, nitrides, mixed oxides, and composites. Over the last decade, the use of ceramic nanostructures has already changed the approach to materials design in many of these applications, by seeking structural control at the atomic level and tailoring of the engineering properties. The articles in this issue review the advantages of nanoceramics, their application in various fields, and the challenges involved in their fabrication.
\end{abstract}

Keywords: bioceramics, consolidation, nanoceramics, nanocomposites, nanostructure, nanotubes, photovoltaics, semiconductors.
Materials scientists today are being challenged to discover, build, control, and test structures whose dimensions range in the nanometer scale and to demonstrate the potential of these nanostructures in scientific, industrial, or medical applications while keeping in mind their potential impact on society. The resulting so-called nanotechnology, which implies the control of matter at the atomic and molecular level, is requiring researchers to work across not only the boundaries of classical scientific disciplines, but also those of other fields, including the social sciences and education. ${ }^{1,2}$ Figure 1 gives a basic scheme of the interdisciplinary character of nanotechnology by showing the overlaps between the science of nanotechnology and various application fields.

As often happens in the early stages of a promising new technology, strong hopes have been placed on nanomaterials to solve all the current problems in many scientific fields, from electronics, optoelectronics, and photonics to energy storage, medicine, and biology. It is indeed true that many nanoscale approaches that have been proposed can in principle provide solutions to difficult scientific problems; successful demonstrations of this have been made in laboratories throughout the world (see References 3-9 for examples). Some applications that have already benefited from nanomaterials and nanotechnology are mentioned in Figure 1. However, a delicate point to address is that the integration processes remains a challenge.

Gaining an understanding of and, ultimately, control over the properties and behavior of a wide range of materials at the nanoscale is now a major theme in materials research. The properties of the resulting products and devices ultimately depend on how the atoms are arranged in the material. For example, atoms in coal can be rearranged to make diamond; atoms in sand can be manipulated (with the addition of a few other trace elements) to make computer chips. As researchers' ability to synthesize materials and fabricate structures at the nanoscale improves, effective characterization becomes a stringent requirement. Although new instruments with enhanced sensitivity and resolution may be necessary, innovative approaches are essential for tackling the problems, addressing the difficulties, and finally reaching solutions that will lead to new products and devices fabricated on the nanoscale.

This issue of MRS Bulletin specifically deals with ceramic nanostructures. Ceramics have been known since the early days of human civilization. The word "ceramic" comes from the Greek keras, meaning horn. In prehistoric times, animal horns were used as containers; later, containers made out of clay were used to store food, water, wine, and oil. Keramica describes the working of the clay into pottery. Observing the hardening of the clay under the hot desert sun may have given our ancestors the idea that clay would of nanotechnology in current industrial

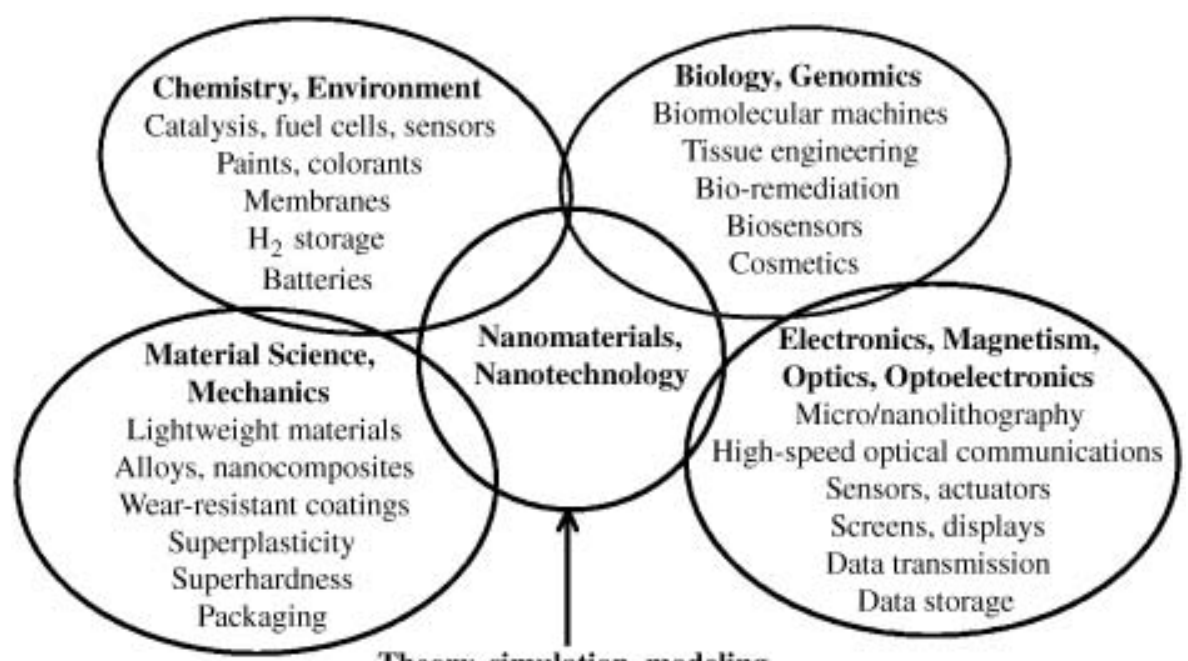

Theory, simulation, modeling

Tools appropriate to nanoscale investigation

Figure 1. Schematic illustration of the interdisciplinary character of nanotechnology, showing the overlaps between the science of nanomaterials and application fields. 
harden even more if subjected to firing. Over the centuries, the technology of ceramic fabrication, at the macroscale at least, evolved to high levels of sophistication and artistry (Figure 2).

Since the beginning of the industrial era in the 19th century, ceramics have been steadily integrated in the fabrication processes for technological applications. Conventional ceramic materials are widely used today in areas ranging from structural to biological applications and in devices as diverse as lasers, semiconductors,
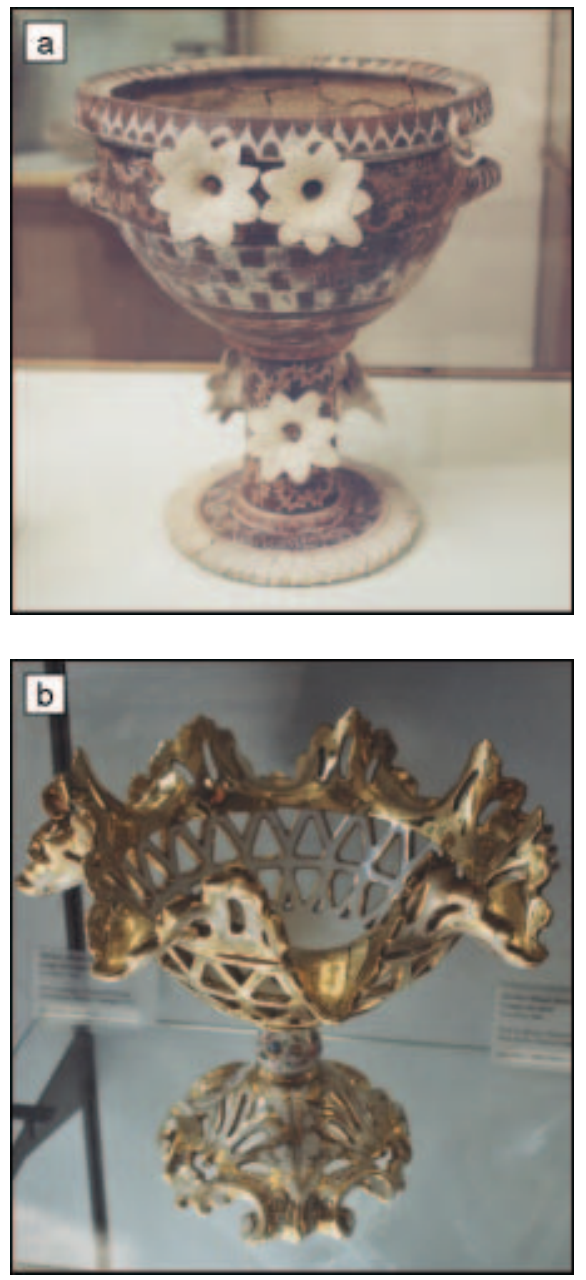

Figure 2. Examples of the high level of sophistication and artistry achieved in ceramic technology on the macroscale over the centuries. (a) Ceramic vase in the Kamares style from Phaestos, Greece, 1900-1700 BC (Archaeological Museum, Heraklion, Greece). Height, approximately $70 \mathrm{~cm}$. (b) Limoges porcelain dish, France, 1834 AD (Adrien Dubouche Museum, Limoges, France.) Height, approximately $30 \mathrm{~cm}$. Note that these two works are separated by an interval of almost 40 centuries.

Photographs courtesy of M.-I. Baraton. sensors, and piezoelectric components. Such materials include oxides, carbides, nitrides, mixed oxides, and metal/oxide composites. Over the last decade, with the successful upscaling of their production, nanoceramics have been revolutionizing traditional materials design in many applications, by the tailoring of their properties via structural control at the atomic level. Some of the advantages of nanoceramics in various fields of application can be highlighted:

- Manufacturing defect-free powder products in all classes of complexity with uniform density;

- Improving the quality and performance characteristics (hardness, plasticity, wear resistance, uniform density, etc.) of sintered ceramic products as a result of their nanostructured formation;

- Reducing the size of electronic devices (transistors), thanks to nanocrystalline gate insulators with higher dielectric capabilities;

- Developing ceramic coatings for greater biocompatibility in medical implants; and

- Using ceramic nanoparticles as drug carriers for site-specific drug delivery.

This issue of MRS Bulletin is aimed at addressing the needs in the field of ceramic nanostructures for industrial applications, as illustrated by several articles contributed by researchers from around the world (United States, Europe, Japan, and Australia). Our intent is to provide examples of the development of nanoceramics and their wide range of applications rather than to give a comprehensive treatment of only one aspect of this extremely active and rapidly growing field of research. Several generations of nanotechnology products are expected to evolve from relatively simple nanostructures for products such as ceramic coatings and hard metals, to active components such as nanoscale transistors, and then to nanosystems with new architectures.

The first article, by Seal et al., illustrates the promises and pitfalls of nanotechnology, which are mostly based upon the ability to produce bulk nanostructured parts exhibiting novel properties at the macroscale. ${ }^{10}$ The authors describe the present state of knowledge of the fabrication and consolidation of so-called nanocomposites, defined here as a class of materials in which at least one of the constituents is in the nanometer domain. Special emphasis is placed on some encouraging results in the plasma-forming of bulk parts. Future challenges for developing methods for consolidating large nanocomposites while retaining their nanostructure are also highlighted.
The second article, by Kuntz et al., is a complement to the first and focuses on nanocrystalline ceramic composites (defined here as having both phases in the nanometer domain) specifically designed for applications requiring improved fracture toughness. While the models and theory of toughening mechanisms in microcrystalline composites are well developed, the same cannot be said for their nanocrystalline counterparts. The difficulty in producing fully consolidated ceramic composites that retain a nanocrystalline structure is the main hurdle to thorough investigations in this area. Thus, much of the research on "nanocomposites" has actually been on materials with microcrystalline matrices and nanometric secondary phases. This article focuses on the production and testing of composites that have nanocrystalline structures for both the matrices and the secondary phases. The microcrystalline mechanisms and current results are reviewed.

Nanoceramics in biomedical applications constitute the subject of the third article, by Ben-Nissan. An improved understanding of the interactions at the nanoscale between the bioceramics in human implants and the hard or soft tissues in the body could contribute significantly to the design of new-generation prostheses and postoperative patient management strategies. Overall, the benefits of advanced ceramic materials in biomedical applications have been universally accepted, specifically in terms of their strength, biocompatibility, hydrophilicity, and wear resistance in articulating joints. The continuous development of new-generation implants utilizing nanocoatings with novel nanosensors and devices is pertinent for better biocompatibility and improved well-being and longevity for the patients. This article gives a short overview of bioceramics and reexamines key issues of concern for the processing and application of nanoceramics as biomaterials.

Further application of nanoceramics in the biomedical arena is dealt with in the article by Kriven et al. The authors present results showing that bioresorbable nanoceramics can be used for drug delivery. For example, biofunctional molecules can be incorporated between hydroxide layers of clay nanoparticles to form bio-layered double hydroxide (LDH) nanohybrids. Once introduced into the cell, the LDH is purposely removed slowly by dissolving it in a pH-controlled environment, and the encapsulated biomolecules are thus released inside the cell. Preliminary safety studies with rats showed that there were no systemic effects as demonstrated by clinical chemistry and histopathology. 
In vitro studies indicated that pristine LDH nanohybrid material had no cytotoxic or growth-inhibitory effects. From the pharmacokinetic in vivo studies, the possibility of using the bio-LDH nanohybrids as a device for slow-release intravenous drug administration can be suggested.

The fifth article, by Golberg et al., presents nanotubular structures in the B-C-N ceramic system that represent an intriguing alternative to conventional carbon nanotubes (CNTs). Because of the ability to widely vary nanotube chemical composition within the B-C-N ternary phase diagram and to change the stacking of $C$-rich or BN-rich tubular shells in multiwalled structures, a wide horizon opens up for tuning nanostructure electrical properties. Pure CNTs are metals or narrow-bandgap semiconductors, depending on the helicity and diameter of the nanotubes, whereas $\mathrm{BN}$ nanotubes are insulators with a $\sim 5.0 \mathrm{eV}$ gap independent of these parameters. Thus, the relative $\mathrm{B} / \mathrm{C} / \mathrm{N}$ ratios and/or $\mathrm{BN}$-rich and $\mathrm{C}$-rich domain spatial arrangements, rather than tube helicity and diameter, are assumed to primarily determine the B-C-N nanotube electrical response. This characteristic is highly valuable for nanotechnology because while tube diameter and helicity are currently difficult to control, continuous doping of $\mathrm{C}$ with $\mathrm{BN}$, or vice versa, proceeds relatively easily due to the isostructural nature of layered $\mathrm{C}$ and $\mathrm{BN}$ materials. In this article, recent progress in synthesis, microscopic analysis, and electrical property measurements of a variety of compound nanotubes in the ceramic B-C-N system is documented and discussed.

Besides structural materials, nanoceramics are intended to play a major role in photovoltaic devices, representing a potentially huge market. In the last article, Brabec et al. consider nanostructures, nanoparticles, and nanoceramics for novel photovoltaic devices and show that by controlling the morphology of organic and inorganic semiconductors on a molecular scale, nanoscaled $p-n$ junctions can be generated in a bulk composite. Such a composite is typically called a "bulk heterojunction composite" and can be considered as one virtual semiconductor combining the electrical and optical properties of the single components. Solar cells constitute one attractive application for bulk heterojunction composites. The $p$ type semiconducting class encompasses conjugated polymers or oligomers, while for $n$-type semiconductors, inorganic nanoparticles as well as organic molecules are investigated. Due to the solubility of the single components, production relies on printing techniques.
The nonexhaustive examples of ceramic nanostructures presented in this issue demonstrate that multidisciplinary research in nanotechnology is not an option, but rather a necessity. It becomes therefore critical and urgent that researchers from various areas share their expertise and develop a common language. This will ensure faster integration of nanotechnology in industry, in the best interests of society.

\section{References}

1. N. Lane, J. Nanopart. Research 3 (2-3) (2001) p. 95-103.

2. Roco and W.S. Bainbridge, J. Nanopart. Research 4 (4) (2002) p. 281.

3. H.O. Jacobs, A.R. Tao, A. Schwartz, D.H. Gracias, and G.M. Whitesides, Science 296 (2002) p. 323.

4. S. Zhang, Nat. Biotechnol. 21 (10) (2003) p. 1171.

5. Y. Cui and C.M. Lieber, Science 291 (2001) p. 1118

6. Y. Huang, X. Duan, Q. Wei, and C.M. Lieber, Science 291 (2001) p. 630.

7. G. Skandan, Nanostruct. Mater. 5 (1995) p. 111.

8. I.V. Alexandrov, Y.T. Zhu, T.C. Lowe, R.K. Islamgaliev, and R.Z. Valiev, Nanostruct. Mater. 10 (1998) p. 45.

9. A. Agarwal, T. McKechnie, and S. Seal, J. Thermal Spray Technol. 12 (3) (2003) p. 350.

10. A. Sweeney, P. Vaidyanathan, and S. Seal, Bull. Sci. Technol. 23 (4) (2003) p. 236
Sudipta Seal, Guest Editor for this issue of MRS Bulletin, is an associate professor of materials science and engineering in the Advanced Materials Processing and Analysis Center (AMPAC) and the Department of Mechanical, Materials, and Aerospace Engineering at the University of Central Florida (UCF) in Orlando. He has served as a nanoinitiative coordinator for UCF since 2001. Seal's research has focused on the synthesis, characterization, and application of nanostructured oxide and metallic materials and associated surfaceengineering-related phenomena. His expertise lies in sol-gel and microemulsion-synthesized nanomaterials for coat- ings for high-temperature and room-temperature gas sensors and bulk nanostructure fabrication of lightweight, high-strength materials using plasma-spray forming. His most recent work has involved developing regenerative oxide nanomaterials in collaboration with a microbiologist for multifaceted biological applications in aging and in the prevention of cell dysfunction and cardiovascular disease.

Seal received his $\mathrm{PhD}$ degree in 1996 from the University of Wisconsin and an MS degree from the University of Sheffield (United Kingdom). He received a BTech degree from the Indian Institute of Technology (IIT) in metallurgical and materials engineering in 1990. He joined the Advanced Light Source at Lawrence Berkeley National Laboratory, University of California-Berkeley, as a postdoctoral fellow in materials science, synchrotron radiation $\mathrm{x}$-ray photoelectron spectroscopy, and microscopy of advanced materials. In the fall of 1997, he joined the faculty at UCF. He is also an adjunct faculty member in the Biomolecular Science Department at UCF.

Seal serves on the editorial boards of the Journal of Nanoscience and Nanotechnology, Sensors Letters, and Reviews in Advanced Materials; the review board of Metallurgical Transactions; and is chair of the Surface Engineering sector for JOM. Starting in 2004, he will

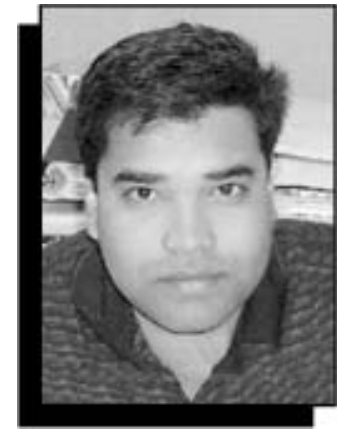

Sudipta Seal

be an associate editor for the Journal of Vacuum Science and Technology. Seal has published more than 135 peer-

reviewed journal papers, edited books and book chapters, and delivered more than 150 invited lectures and research presentations in the United States and abroad. He has organized symposia for TMS,

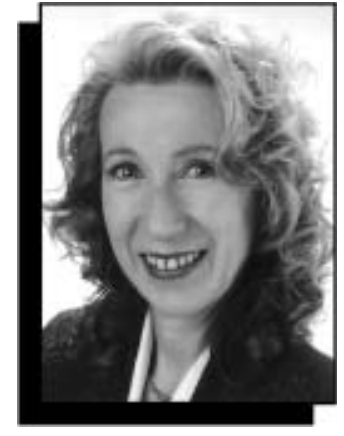

Marie-Isabelle Baraton

ASM International, and ECS on surface engineering and nanotechnology and recently organized a workshop on nanotechnology (Crete, Greece) sponsored by National Science Foundation. He is the recipient of the 2002 ONR Young Investigator Award, the 2003 Distinguished ASM-IIM Lecturer Award from ASM International, and 
the 2004 Japan Society for the Promotion of Science (JSPS) fellow award. He is a member of ASM International, TMS, the Materials Research Society, the Electrochemical Society, and the American Vacuum Society (AVS).

Seal can be reached at AMPAC, MMAE, University of Central Florida, 4000 University Blvd.,

Eng 381, Orlando, FL 32816, USA; tel. 407-8821119 or $407-823-5277$, fax 407-882-1156, and e-mail sseal@mail.ucf.edu and sseal@pegasus.cc.ucf.edu.

\section{Marie-Isabelle Baraton,} Guest Editor for this issue of MRS Bulletin, is a senior scientist in the Department of Ceramics at the University of Limoges, France, where she leads research on nanostructured materials. Her current research interests include the physicochemistry of nanomaterial surfaces (metal oxides and nonoxide ceramics such as $\mathrm{SiC}, \mathrm{AlN}, \mathrm{GaN}$, and $\mathrm{Si}_{3} \mathrm{~N}_{4}$ ), as well as theoretical (ab initio calculations) and experimental studies of chemical reactions at gas/nanomaterial interfaces. Her research work finds applications in the dispersion of nanoparticles in polymeric matrices for microelectronics, semiconductor passivation and surface functionalization, gas sensing, self-assembled layers, and coatings. Baraton received her $\mathrm{PhD}$ degree in physics in 1971 and her doctorate in science in 1979 from the University of Limoges. In 1986-1987, she obtained a NATO grant to conduct fundamental research on infrared surface characterization of nano-sized powders and on LangmuirBlodgett films in the
Department of Chemistry at the University of Ottawa and in the Lash Miller Laboratories at the University of Toronto. Baraton has co-authored over 100 refereed papers, communications, and book chapters, including review articles on FTIR surface characterization of nanoparticles. She is the editor of a recently published book on the functionalization of nanoparticles. As the leader of European consortia comprising industries and universities funded by the European Commission, Baraton has coordinated work on novel gas sensors based on nanomaterials for air quality monitoring. She also promoted scientific exchanges on an international basis as director for NATO countries in the organization of a NATO Advanced Study Institute in Kiev, Ukraine, in 2000.

Baraton is a member of the Materials Research Society, the American Chemical Society, and the European Materials Research Society. She organized a symposium on nanomaterials in 1997 for MRS, has served as an MRS Bulletin volume organizer, and was a meeting chair for the 2002 MRS Fall Meeting. Baraton is also chair of the MRS International Relations Committee.

Baraton can be reached at SPCTS-UMR 6638 CNRS, Faculty of Sciences, 123 Avenue Albert Thomas, F-87060 Limoges, France; tel. 33-555-45-7348, fax 3355-77-8100, and e-mail baraton@unilim.fr.

Arvind Agarwal is an assistant professor in the Department of Mechanical and Materials Engineering at Florida International University

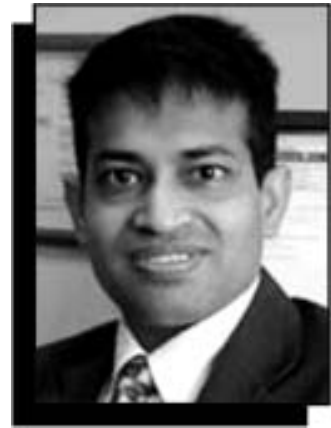

Arvind Agarwal

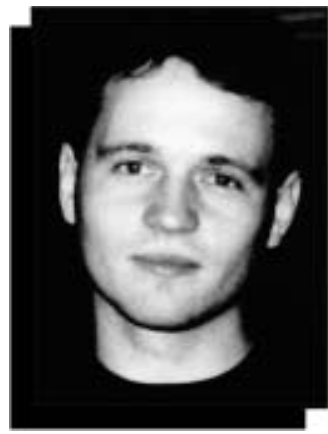

Pavel Dorozhkin

(FIU) in Miami. He received his BS and MS degrees from the Indian Institute of Technology (IIT), Kanpur, and his PhD degree from the University of Tennessee, Knoxville, in 1999.

Agarwal worked as a materials scientist at Plasma Processes Inc. (Huntsville, Ala.) from December 1999 to November 2002. He also served as adjunct professor at the University of Alabama, Huntsville. Agarwal joined the FIU faculty in November 2002. His current research interests include processing of bulk nanostructured materials, near-net-shape processing and rapid prototyping, thermal spray techniques, and ultrahigh-temperature ceramic coatings and composites. His earlier research was focused on laser materials processing and synthesis and pulsed electrode surfacing. Agarwal has published more than 40 research papers and co-

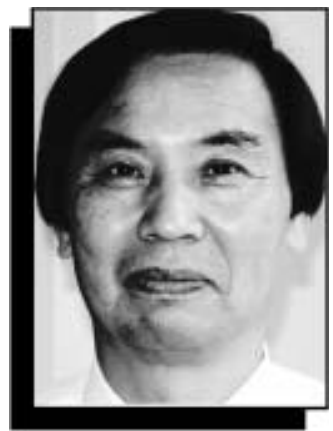

Yoshio Bando

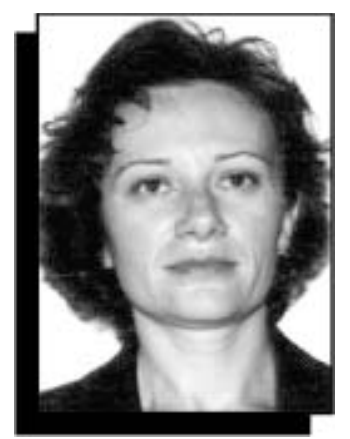

Petya Georgieva

edited two books. He serves on the international board of review for the Journal of Materials Engineering and Performance. He has organized symposia for TMS and ASM International on surface engineering. $\mathrm{He}$ is the co-organizer for the Surfaces and Interfaces in Nanostructured Materials symposium to be held at the 2004 TMS meeting and is the primary organizer and editor of the proceedings of the Surface Engineering in Materials Science III symposium for the 2005 TMS meeting. He is a member of ASM International, TMS, and the Materials Research Society.

Agarwal can be reached by e-mail at agarwala@fiu.edu.

Yoshio Bando is a director of the Advanced Beam Analysis Group of the Advanced Materials Laboratory and a director-general of the International Center for Young Scientists within

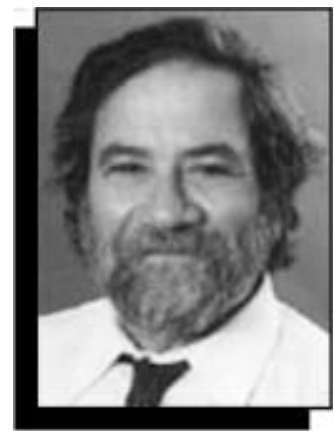

Besim Ben-Nissan

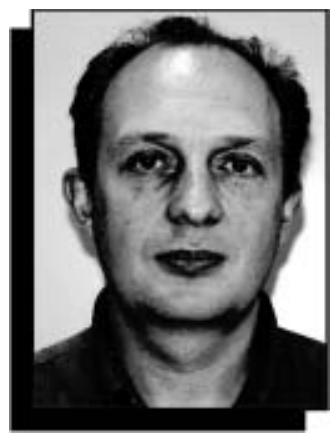

Dmitri Golberg

the National Institute for Materials Science (NIMS) in Tsukuba, Japan. He is also a professor at the University of Tsukuba. His current research concentrates on preparation, electron microscopy, and property analysis of inorganic nanostructures, including nanotubes, nanowires, nanocones, nanoparticles, and nanobelts.

Bando received his $\mathrm{PhD}$ degree in physics from Osaka University in 1975 and joined NIMS the same year. From 1979 to 1981, he worked as a visiting researcher at Arizona State University. Bando has authored or co-authored more than 200 original papers and over 50 patents. $\mathrm{He}$ is a recipient of several awards, including Best Paper of the Year Awards $(1992,1999)$ and the Seto Award (1994) from the Japanese Society for Electron Microscopy and academic awards from the Science and Technology Agency of 


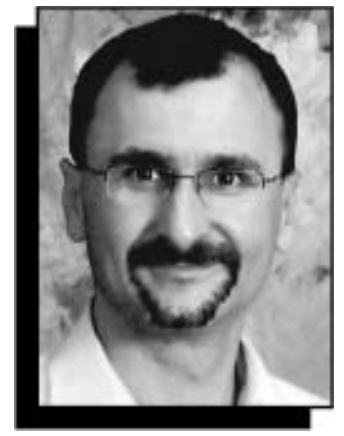

Christoph J. Brabec

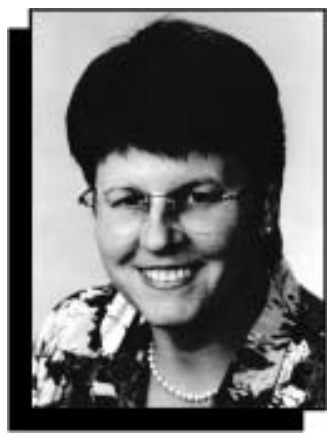

Waltraud M. Kriven

Japan (1994) and the Japanese Ceramic Society (1997). He has also served as an organizer or coorganizer for various international conferences and symposia. He is currently president of the Japanese Electron Microscopy Society's Kanto branch and a member of the editorial boards for the Journal of Electron Microscopy and the Journal of Nanoscience and Nanotechnology.

Bando can be reached by e-mail at bando. yoshio@nims.go.jp.

Besim Ben-Nissan is an associate professor in the Department of Chemistry, Materials, and Forensic Science at the University of Technology, Sydney, in Australia. His expertise is in advanced ceramics and biomaterials, specifically hydroxyapatite (including the conversion of Australian corals to hydroxyapatite) and modeling of biomedical implants. Over the last

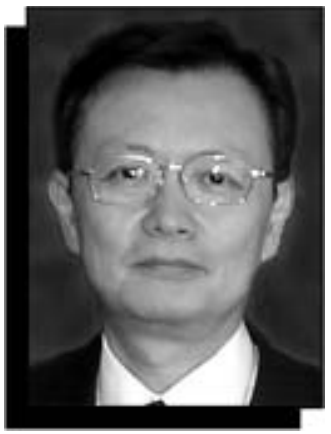

Jin-Ho Choy

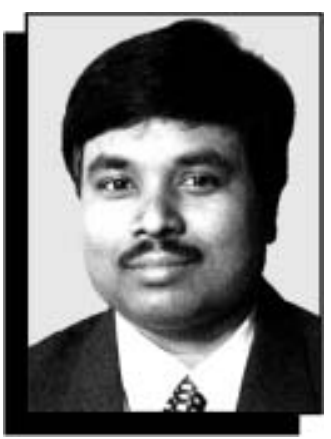

Suresh C. Kuiry

two decades, he has contributed to the production and analysis of advanced sol-geldeveloped thin films, biomedical materials, material structures and modeling (using finite element analysis), bone graft production, biocomposites, biomechanics (including investigative research on jaw bone, knee, and hip joints), reliability, and implant design (modular zirconia ceramic knee prostheses, femoral head and taper stresses, and bionic eyes).

Ben-Nissan holds advanced degrees in metallurgical engineering and ceramics engineering (MSc) and mechanical engineering jointly with biomedical engineering $(\mathrm{PhD})$ from the University of New South Wales. He has published more than 170 papers in journals, books, and proceedings. He was awarded the Australasian Ceramic Society Award in 2000 for his sustained

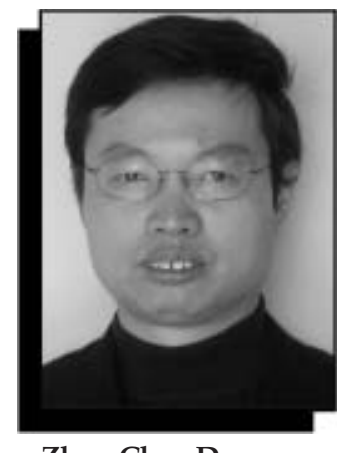

Zhen-Chao Dong

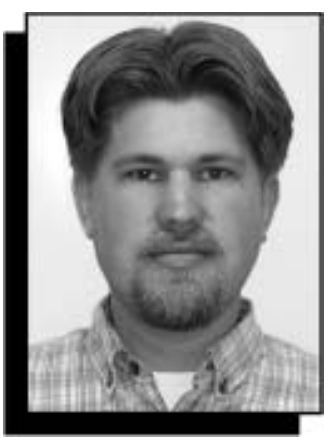

Joshua D. Kuntz

contribution to ceramics research and development and education in Australia.

Ben-Nissan can be reached by e-mail at b.ben-nissan@uts.edu.au.

Christoph J. Brabec is a project leader at Siemens Corporate Technology in Erlangen, Germany, where he is involved in the development of organic semiconductor devices. During his $\mathrm{PhD}$ studies (1995), he investigated the rheology of polymer melts with respect to molar mass correlations. In 1996, he joined the group of Alan Heeger at the University of Santa Barbara for a sabbatical, and he continued to work on the optoelectronic properties of organic semiconductors later on as an assistant professor at the University of Linz, Austria. In 1998, he became a senior scientist within the Christian Doppler Laboratory for organic solar cells, which he left in 2001 to join Siemens. $\mathrm{He}$ is the author or coauthor of more than 100 papers and 30 patents and finished his habilitation in physical chemistry in 2003.

Brabec can be reached by e-mail at christoph. brabec@siemens.com.

Jin-Ho Choy has been a professor in the School of Chemistry and Molecular Engineering at Seoul National University since 1981 . He is an advisory editorial board member of Chemistry of Materials, the Journal of Solid-State Chemistry, the Materials Research Bulletin, SolidState Sciences, and the Bulletin of the Korean Chemical Society. He received two PhD degrees: in chemistry, from the Institute for Inorganic Chemistry at the University of Munich, Germany (1979); and in materials engineering, from the Research Laboratory of Engineering Materials at the Tokyo Institute of Technology, Japan (1993). In 1980, he was a postdoctoral fellow in the Inorganic Chemistry Laboratory at the University of Oxford, developing semiconductor electrodes for water electrolysis. Choy was also a visiting scientist at the Solid State Chemistry Laboratory (LCS) at Bordeaux University, France, from 1984 to 1985 , where he undertook synthesis studies of new functional materials under extreme conditions. Although Choy's research area is highly multidisciplinary, the main subject of his research is the exploration of novel nanohybrid materials by means of intercalation reactions. His current research is focused on drug and gene delivery systems based on inorganic nanomaterials. He has published 390 papers in international scientific journals, written or edited six books, holds 30 patents, and was granted a National Science Award in chemistry (2000). He is a permanent fellow of the Korean Academy of Science and Engineering.

Choy can be reached by e-mail at jhchoy@ snu.ac.kr.

Zhen-Chao Dong is a senior researcher in the Nanomaterials Laboratory at the National Institute for Materials Science (NIMS) in Tsukuba, Japan. His research interests focus on the fabrication and characterization of nano- and atomic-scale structures as well as molecular optoelectronics with an emphasis on tunnelingelectron-induced fluorescence from single molecules.

Dong obtained a BS degree in chemistry from Sichuan University (1983), a MS degree from Xiamen University (1987), and a PhD degree at the Fujian Institute of Research on the Structure of Matter, Chinese Academy of Sciences (1990). After postdoctoral research at Iowa State University, he joined the National Research Institute for Metals in 1996 and was promoted to senior researcher in 1998. He was selected for the "Hundred People Program" by the Chinese Academy of Sciences in 1999 and has authored more than 100 publications.

Dong can be reached by e-mail at dong.zhenchao@nims.go.jp.

Pavel Dorozhkin is a researcher at the Institute of Solid-State Physics in 
Chernogolovka, Russia, where he is finalizing his $\mathrm{PhD}$ degree in the field of optical studies of individual semimagnetic quantum dots. He graduated with an MS degree in physics from the Moscow Institute of Physics and Technology in 1999. He was a research fellow at the National Institute for Materials Science (NIMS) in

Tsukuba, Japan, from 1998 to 2000 and a visiting researcher at NIMS in 2001 and 2002. His research there concentrated on low-energy electron microscopy, nanomanipulation, transport, and field-emission studies of individual nanotube ropes of various types. $\mathrm{He}$ is a co-author of 14 journal papers.

Dorozhkin can be reached by e-mail at pdorozh@issp.ac.ru.

Petya Georgieva is a $\mathrm{PhD}$ student in materials science and engineering at the University of Central Florida in Orlando. Her area of expertise is in microstructural characterization of metal/ ceramic nanostructures and consolidation of bulk nanocomposites using computational modeling. She received master's degrees in chemistry and physics from Sofia University, Bulgaria, in 1998. Currently, she works at the Advanced Materials Processing and Analysis Center at UCF on the development of industrially important nanoparticles and their consolidation into nanostructured bulk components. Her primary research interests include microstructural characterization of plasma-sprayed materials and near-net-shape forming techniques for advanced materials and components. Georgieva was recently awarded an NSF fellowship from Northwestern University to attend a workshop on Nanoscale Design of Materials. Georgieva can be reached by e-mail at pmgeorgieva@aol.com.

\section{Dmitri Golberg is a} senior researcher at the Advanced Materials

Laboratory within the

National Institute for

Materials Science (NIMS)

in Tsukuba, Japan. His

current research interests

focus on synthesis, ad-

vanced electron micros-

copy (e.g., high-resolution and energy-filtered TEM), and physical property measurements of inorganic nanotubes, in particular, B-C-N and BN nanotubes. He also studied shape-memory alloys as a visiting researcher at the University of Tsukuba (1993) and single crystals of intermetallic compounds as a guest scientist at the Max-Planck-Institut für Eisenforschung in Duesseldorf (1994) and the National Institute for Metals in Tsukuba (1996).

Golberg received his $\mathrm{PhD}$ degree in solid-state physics from Bardin Central Research Institute for Ferrous Metallurgy (Moscow) in 1990, where he worked for more than 10 years before moving to Japan in 1993. He received the Best Paper of the Year Award in Materials Science from the Japanese Society for Electron Microscopy in 1999. Golberg is the author or co-author of more than 90 original papers, 30 patents, and over 45 presentations at international scientific forums. He has also authored chapters in books including Electron Microscopy

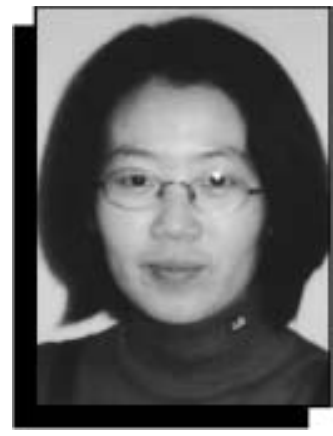

Seo-Young Kwak

of Nanotubes (Kluwer

Academic Press, 2003).

Golberg can be reached

by e-mail at golberg.

dmitri@nims.go.jp.

Waltraud M. Kriven is a professor in the Department of Materials Science and Engineering at the University of Illinois at Urbana-Champaign.

Her research areas in-

clude the design and

fabrication of structural ceramic composites; mechanisms of martensitic transformations, ferroelasticity and ferroelastic transformations in ceramics; microstructure characterization by electron microscopy (SEM, TEM, EDS,

HVEM, XPS); and bioresorbable nanoceramics for gene and drug delivery. Her undergraduate degrees are in physical and inorganic chemistry with a minor in biochemistry from Adelaide University in South Australia and she received her $\mathrm{PhD}$ degree in 1976 in solid-state chemistry from Adelaide. Kriven spent three years (1977-1980) jointly at the University of California at Berkeley and Lawrence Berkeley Laboratory. For almost four years (1980-1983),

Kriven was a visiting scientist at the Max

Planck Institute in

Stuttgart, Germany, where she undertook

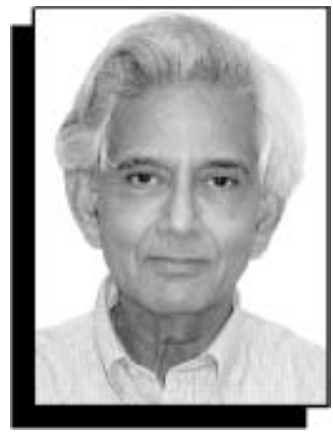

Amiya K. Mukherjee

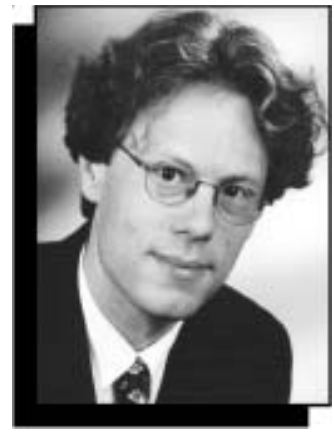

Thomas Nann high-voltage electron microscopy studies of transformation toughening in zirconia ceramic composites. She has written or co-authored more than 210 research publications and Coauthored three US patents, and she is a fellow of the American Ceramic Society (ACS). She is currently vice chair of the Engineering Ceramics Division of ACS.

Kriven can be reached by e-mail at kriven@ uiuc.edu.

Suresh C. Kuiry is a research associate at the Advanced Materials

Processing and Analysis Center within the University of Central Florida in Orlando. His present interests include the synthesis of nanomaterials for high-temperature coatings, gas sensing, and biological applications; consolidation of ceramic nanocomposites; metal-filled carbon nanotubes; chemicalmechanical planarization; high-temperature oxidation of metals and alloys; and the thermodynamics of point defects in semiconducting materials. He received his $\mathrm{PhD}$ degree in metallurgical and materials engineering from the Indian Institute of Technology (IIT), Kharagpur, in 1996. Prior to joining the Uni- versity of Central Florida, he worked as the research manager for Mukand Ltd. in Mumbai, India. Kuiry received the National Young Metallurgist award from the Indian Ministry of Steel and Mines in 1998 for his outstanding research in materials engineering. $\mathrm{He}$ is also a recipient of a Gold Medal from the University of Burdwan. Kuiry has published more than 40 articles in international journals, authored several book chapters, and presented more than 50 papers at international conferences.

Kuiry can be reached by e-mail at sckuiry@ pegasus.cc.ucf.edu.

Joshua D. Kuntz is a $\mathrm{PhD}$ candidate in materials science and engineering at the University of California, Davis. His dissertation research is in the area of synthesis, processing, and characterization of ceramic nanocomposites designed for improved fracture toughness. His other research interests include the development of metallically conducting ceramic composites and novel processing techniques for powder consolidation and superplasticity in nanocrystalline ceramics and metals. Kuntz earned a BS degree in materials science and engineering 


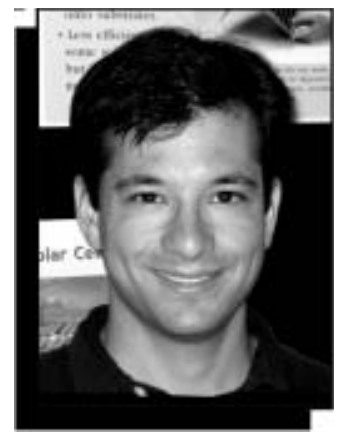

Sean E. Shaheen

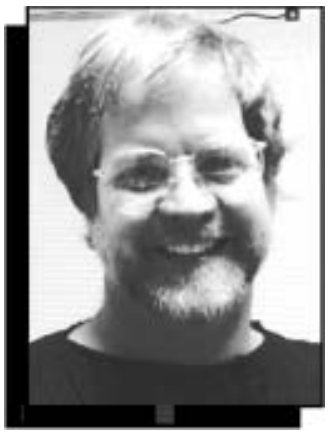

Matthew A. Wallig

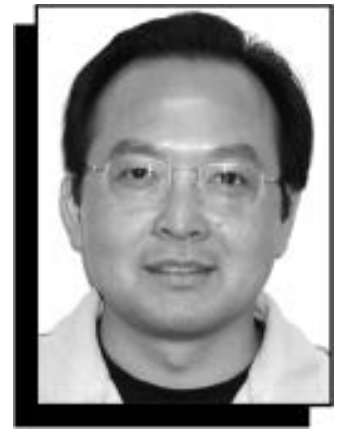

Guo-Dong Zhan from UC Davis. Kuntz is a member of AcerS, MRS, ASM International, and TMS and has received several academic awards, including the John Dorn Award from ASM.

Kuntz can be reached by e-mail at jdkuntz@ ucdavis.edu.

Seo-Young Kwak is a postdoctoral research associate in the Department of Materials Science and Engineering at the University of Illinois at Urbana-Champaign. She received her MA and $\mathrm{PhD}$ degrees in inorganic chemistry from Seoul National University in 1998 and 2001, respectively. She has eight years of experience in the fields of two-dimensional inorganic materials and bio-inorganic nanohybrid materials as drug and gene delivery carriers. Her major research topics are bio-inorganic materials as nonviral vectors; the design and development of layered compounds applicable to fields such as photochemistry and pharmaceutical chemistry; exploration of new ceramic materials, their structural study, and physicochemical characterization; and nanopore, nanoparticle, and nanocomposite chemistry.

Kwak can be reached by e-mail at seokwak@ uiuc.edu.
Amiya K. Mukherjee is a professor of materials science and engineering at the University of California, Davis. His current research interest is in the area of synthesis, processing, and characterization of nanocrystalline metals and ceramics and the study of their physical and mechanical behavior. After completing his $\mathrm{PhD}$ (DPhil) degree at Oxford University in England, he first joined Lawrence Berkeley National Laboratory as a research metallurgist and subsequently the faculty of the College of Engineering at UC Davis. He has authored or coauthored more than 400 papers with his students and postdoctoral research associates. He is a fellow of ASM and has received the University of California Citation, the Albert Easton White Distinguished Award (ASM International), the Pfiel Medal from the Institute of Metals (Great Britain), and the Alexander von

Humboldt Award.

Mukherjee can be reached by e-mail at akmukherjee@ ucdavis.edu.

Thomas Nann is scientific assistant to the Chair for Sensors at the Institute for Microsystem Technology of the University of Freiburg, Germany, and leader of the
Nanoscience Group at the Freiburg Materials Research Center (FMF). His scientific interests are particularly focused on the preparation, derivatization, and application of nanocrystals for electro-optics and bioanalytics. Nann studied chemistry at the Universities of Stuttgart and Freiburg and received his diploma in 1994 from the University of Freiburg. During his PhD studies, he worked on digital simulation of kinetic processes at microelectrodes and the connected mechanisms.

Nann can be reached by e-mail at thomas.nann@ fmf.uni-freiburg.de.

Sean E. Shaheen is a researcher at the National Renewable Energy Laboratory in Golden, Colo., where he works on novel device designs for organic and nanostructured oxide-based solar cells. His interests include charge transport and carrier dynamics in disordered media, interface physics, computational-chemistry-aided molecular design, and simulations and modeling of organic and oxide composite photovoltaic devices. He received his $\mathrm{PhD}$ degree in physics from the University of Arizona (1999) and performed postdoctoral re- search at the University of Linz, Austria, as a Lise Meitner postdoctoral fellow. His research work extends to complex dynamical systems, information processing in biological systems, and decisionmaking in uncertain environments.

Shaheen can be reached by e-mail at sshaheen@nrel.gov.

Matthew A. Wallig is a professor of comparative pathology in the Department of Veterinary Pathobiology at the University of Illinois at Urbana-Champaign. He is also a diplomate of the American College of Veterinary Pathologists. Wallig received his BA degree in zoology from the University of Wyoming in 1976 and his DVM degree from the University of Minnesota in 1981. His residency and $\mathrm{PhD}$ degree in pathology were completed in 1987 at Colorado State University. In 1987, he joined the faculty at the University of Illinois. In 1991, he became a fellow of the American Association for the Advancement of Science. Wallig has written or co-authored more than 60 peer-reviewed research publications in nutritional sciences, toxicology, pathology, feed science, chemistry, and clinical medicine. His research areas include defining synergistic interactions among phytochemicals in cruciferous plants in the prevention of cancer, studying the anticancer effects of various phytochemicals in fruits and vegetables, biomarkers of malignancy in various forms of cancer, and the role of apoptosis in various clinical conditions associated with oxidant stress.
Wallig can be reached by e-mail atmawallig@ uiuc.edu.

Guo-Dong Zhan is a research associate at the University of California, Davis. His work is focused on the synthesis, processing, and characterization of nanostructured materials including ceramics, metals, and metallic glasses and the understanding of the fundamental mechanisms of sintering, toughening, superplasticity, creep, fatigue, and fracture of such materials. Zhan received his $\mathrm{PhD}$ degree in materials science at Huazhong University of Science and Technology, China, in 1994 while researching the processing and mechanical behavior of advanced ceramic composites. He then worked for two years at the Shanghai Institute of Ceramics, where he advanced to the level of associate professor. In 1996, Zhan was invited to be a visiting scholar by Queen Mary University of London. He then headed to Japan, where he spent four years, first as an STA Fellow at the National Institute for Research in Inorganic Materials and then as a staff scientist at the National Institute for Materials Science. Zhan is the author or co-author of more than 70 technical publications in peerreviewed journals and holds several U.S. patents. Zhan has received various awards and is a member of ACerS, MRS, and TMS.

Zhan can be reached by e-mail at gzhan@ ucdavis.edu.

The Materials Gateway

wWw.mis.org 